\title{
AUTOMATED DECISION SUPPORT FOR THE EMERGENCY MANAGER
}

\author{
Dr. John D. Hwang and Louis A. Wofsy* \\ Federal Emergency Management Agency \\ Washington, D.C. 20472
}

\begin{abstract}
Today's emergency managers are required to plan for a wide spectrum of emergencies and to implement these plans under severe time constraints. The traditional, manual methods of managing emergencies do not provide the decision makers with the support they need in today's environment. A number of automated tools have been developed to support various aspects of emergency management but none of them provide fully automated support to the entire spectrum of hazards. The Federal Emergency Management Agency (FEMA) is developing a single architecture to support all phases of emergency response over the full spectrum of hazards. This system will enhance and supplement existing capabilities from FEMA's "toolbox," to perform the complete mission.
\end{abstract}

\section{FEMA AND EMERGENCY MANAGEMENT}

\section{A. FEMA's Mission}

The mission of the Federal Emergency Management Agency (FEMA) is to develop emergency management programs and to coordinate civil agency response to natural disaster emergencies and extraordinary situations in order to protect the civilian population and resources of the nation. Since its creation in 1978, FEMA has developed a wide range of computerized applications and associated databases, commonly denoted as "tools," to assist decision makers in responding to the full range of natural, technological, and national security-related emergencies. These tools have been designed to support many aspects of emergency management activities in planning and coordinating the four phases of emergencies: mitigation, preparedness, response, and recovery. In addition, they provide accurate, timely emergency information for dissemination to the media, the public, and others.

\section{B. Emergency Phases}

Mitigation: The mitigation phase of emergency preparedness deals with planning, policy decisions, regulations, etc., that are designed to anticipate potential emergency situations and either prevent them or limit their harmful consequences. Examples of mitigation actions include engineering solutions, e.g., earthquake-resistant

\footnotetext{
* This paper has been prepared by these authors in their individual capacities, and the views expressed herein are not necessarily those of the Federal Emergency Management Agency.
}

buildings; regulatory actions, e.g., building codes; and public education concerning potential risks.

Preparedness: FEMA's Integrated Emergency Management System operational model defines preparedness as "any activity that develops operational capabilities for responding to an emergency." This includes developing plans for such things as emergency operations and resource allocation as well as management and communications frameworks within which the plans will be executed. Education and training are vital to this phase, with tests and exercises being one of the primary means of accomplishing this objective.

Response: The response phase begins prior to the actual emergency situation in cases where some form of warning is available, e.g., hurricanes, and continues through the emergency period and into the beginning of early recovery activities. The purpose of this phase is to limit the adverse effects of the emergency and protect lives and property.

Recovery: The recovery phase of an emergency covers the efforts necessary to provide assistance to the population and to repair damage to physical structures and institutions so that the affected area is returned to a "business as usual" status. Recovery usually strives to return the affected area to the condition that existed prior to the emergency.

\section{Integrated Emergency Management Concept}

FEMA is moving in the direction of an integrated emergency management concept as shown by the recently published Federal Response Plan (for Public Law 93-288, as amended). This plan supersedes previous hazard-specific Federal disaster response plans, i.e., the Plan for Federal Response to a Catastrophic Earthquake, signed by 25 departments and agencies in 1987. This plan was broadened to an overall federal natural disaster response plan following the 1989 Hurricane Hugo and Loma Prieta earthquake emergencies. The current Federal Response Plan (FRP) has been further expanded following Desert Shield/Desert Storm to address both natural disasters and man-made emergencies

The introductory section of the FRP states that it "is designed to address the consequences of any disaster or emergency situation in which there is a need for Federal response assistance under the authorities of the Stafford Act." It is applicable to natural disasters, such as earthquakes, hurricanes, typhoons, tornadoes and volcanic eruptions; technological emergencies involving radiological or hazardous material releases; and other significant events.

The FRP has been signed by 28 Departments and agencies that will carry out their responsibilities through 12 Emergency Support Functions (ESFs). Each ESF specifies a 
primary Department or agency, with others designated to provide support as appropriate. These ESFs constitute the primary means by which federal assistance will be provided to the states, with the overall effort being directed by a Federal Coordinating Officer.

\section{Situation Assessment}

The key to providing appropriate emergency response is Situation Assessment -- the ability to collect, analyze, and display information so that decision makers can be presented with options and recommendations in a timely fashion. Situation Assessment is an interactive, time-constrained, multi-layered function requiring an information system that can gather and assess both current and historical data and provide viable options to a decision maker. The system must be able to provide timely products based on the best information available, supplementing existing information with appropriate models and projections. Situation Assessment is usually an iterative process that continues to receive and validate data throughout the duration of an emergency. It must also have the capability of supporting a decision maker's needs of performing "what if" analyses.

While most often associated with the response phase of emergency preparedness, Situation Assessment supports all four phases. In the mitigation and preparedness phases the data is normally either historical data for situations similar to the one being considered or projected data. The assessment tools, however, are the same as those used during the response phase and are also used to assist in preparation of emergency response plans. The use of Situation Assessment during recovery is an extension of its use during the response phase, with the primary differences being the availability of more accurate data and, usually, a less-compressed time frame for making decisions.

\section{FEMA'S COMPUTERIZED TOOLBOX}

\section{A. What are FEMA's Tools?}

FEMA has a wide variety of tools available for processing, analyzing, and displaying data both from its own databases and from those of other Departments and agencies. These tools range from very specific, single-purpose applications to flexible, multi-purpose, multi-user capabilities. FEMA's primary efforts since the mid-1980's have been directed towards developing multi-purpose tools that can effectively address the needs of all involved Departments and agencies over a wide spectrum of emergency response situations.

Examples of specific applications include the National Flood Insurance Program and the National Fire Incident Reporting systems. FEMA's multi-purpose tools include the following:

Integrated Emergency Management Information System (IEMIS) - a geographical information capability that can provide extremely detailed archived maps.

National Infrastructure Information System (NIIS) -- an integrated collection of databases, situation assessment information systems, expert systems, and computer models designed to provide decision support to emergency managers.

Emergency Resource Management System (ERMS) - an application that can calculate damage to specific resources based on user-specified criteria and damage modeling capability and display resource and damage information graphically to assist in the management of specific resources.

Automated Message Processing System (AMPS) - a system that facilitates communication between FEMA's national and regional sites using personal computers to generate and receive messages.

Computer Assisted Training Support System (CATSS) -interactive personal computer-based lessons that teach the use of various FEMA tools, e.g., ERMS, AMPS, etc., as well as basic lessons on performing various emergency response functions, e.g., damage assessment.

\section{B. A Framework for the Tools}

The above tools, and others, have been introduced over the last ten years or so to automate various parts of emergency management. Most of these have been of use primarily in the preparedness and recovery phases, with some applicability in the mitigation phase. The development of automated tools for use in the response phase is more recent.

FEMA is now addressing the next step in this progression: namely, providing an automated decision support system which brings all of these tools together. This system will provide an emergency manager with automated support for all phases of the full spectrum of emergencies. The architecture for this system is shown conceptually in the following diagram.

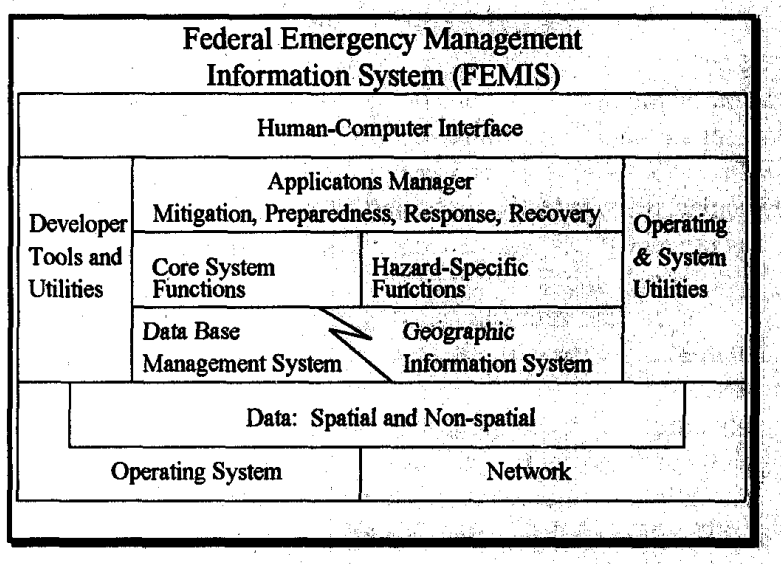

\section{The Design of FEMIS}

FEMIS provides emergency management support through all phases of an emergency by integrating a core set of functions common to all emergencies with the specific set of actions needed for the particular situation. The core functions include: security, communications, direction \& control, alert $\&$ notification, and evacuation.

The Applications Manager is accessed through a humancomputer interface that will be graphical in nature. It

\section{1 .2}


provides the user with the appropriate subset of functions or proper variation of a functional capability for addressing the desired phase of the emergency, both from the core functions and the hazard-specific functions.

The FEMIS functions are supported by a traditional relational database management system (RDBMS). In addition, however, FEMIS will utilize a geographic information system (GIS). The GIS will use the spatial data from the supporting databases to provide a graphical analysis and representation of the situation to the decision maker. This allows the decision maker to rapidly assimilate the material and facilitate the decision-making process. This function makes the GIS a keystone of FEMIS.

In order to support the full spectrum of emergencies, FEMIS will develop a unique Applications Manager and set of hazard-specific functional modules for each hazard. The core functions, RDBMS, GIS, and supporting systems will be the same in all cases. The storage capacity of the system on which FEMIS resides will determine whether all hazards utilize an all-inclusive set of databases, or if the databases will be subdivided to include only the data necessary for a specific hazard.

\section{AN EXAMPLE}

\section{A. Some History of FEMA's GIS Efforts}

In 1981, FEMA initiated the Mobile Exercise Evaluation and Simulation Facility (MEESF) in support of the Radiological Emergency Program. Over time, the MEESF was expanded in scope to handle other types of emergencies and evolved into the Integrated Emergency Management Information System (IEMIS). IEMIS was designed to assist emergency managers and response personnel by providing information management, information exchange, and geographic presentation capabilities for the full range of emergencies covered by Executive Order 12656. The unique achievement of IEMIS is its ability to effectively link federal emergency management models and provide non-proprietary access to public GIS data bases.

The current version of IEMIS integrates various emergency management models, e.g., population evacuation, plume dispersion, and sound propagation. It is used primarily as a planning tool, although it does contain limited operational command and control capabilities, e.g., status boards, checklists, notification lists, message logs, etc.

The PC Risk Estimation System (PCREST) was developed to help state and local, as well as federal, emergency planners to model the effects of nuclear weapons and fallout and display them geographically. PCREST incorporates appropriate models for calculating blast, fallout, and radiation contours over time along with a mapping system to display the model results.

The Emergency Resource Management System (ERMS) provides a means of geographically displaying resources within an area and any damage they are estimated to have sustained from an emergency occurrence. ERMS can be used with any resource database that the user provides and can display multiple resource files at one time while allowing the user to zoom in to see detailed effects or to look at the broad, overall picture. The database needs to include the geographic location of the resource as well as the characteristics necessary to calculate estimated damage effects based on the particular emergency, e.g., hardness, protection factor, etc. Both point (e.g., factory) and vector (e.g., roads) resource files are supported.

The concepts employed in developing the above systems have served as forerunners for the future FEMIS. FEMA sees GIS's playing a very major, positive role in enhancing current systems and providing future automated support systems for emergency decision makers and responders. Such systems will allow all levels of government to respond more quickly, effectively, and efficiently to the entire spectrum of emergencies that face this country today and in the future. This has been applied to FEMA's support for the Army's Chemical Stockpile Emergency Preparedness Program (CSEPP).

\section{B. The Army's CSEPP and FEMA's Automation Support}

The National Defense Authorization Act of 1986 (Public Law 99-145) mandates maximum protection of the public, personnel on Army posts, and the environment during the disposal of unitary chemical weapons. The Chemical Stockpile Emergency Preparedness Program (CSEPP) established by the Army supports State and local organizations in preparing for and protecting the public from the results of an event at military chemical stockpile sites. FEMA has a Memorandum of Understanding with the Army under which FEMA is responsible for developing and implementing an off-post emergency preparedness capability. Development of an automated emergency management information system for the program has been assigned to the CSEPP Automation Subcommittee (CAS) within CSEPP's Joint Steering Committee structure. The Office of Systems Engineering provides the FEMA representative to the CAS

Over the past two years, the CAS has been developing functional specifications for the CSEPP Emergency Management Information System. A pilot project was initiated with the states of Oregon and Utah in 1989 in order to investigate the applicability of Geographic Information System (GIS)-based decision support systems for CSEPP. FEMA's Integrated Emergency Management Information System (IEMIS) was used as the vehicle to demonstrate these concepts. A demonstration was held in Bend, Oregon, in May 1990 and was favorably received. FEMA then proceeded to a second phase specifically designed to provide a prototype system in support of SRFX-91 that was conducted in Tooele, UT in June 1991. The SRFX-91 Pilot Offpost CSEPP Capability (SPOCC) was developed by modifying IEMIS to incorporate an improved user interface, specific CSEPP models, and direction and control functions. SPOCC successfully demonstrated the effectiveness of using such an automated system to improve emergency operations. Following this successful test, the CAS recommended that 
SPOCC be enhanced into a baseline capability to provide a near-term automated solution to the chemical munitions storage site threat while a full-capability system based on the CSEPP functional specifications is developed.

The Joint Steering Committee accepted the CAS recommendation and this Integrated Baseline System (IBS) will be available to all CSEPP sites. The IBS will be used as a basis for planning, database building, training, and assimilation of automation into CSEPP emergency operations. Battelle Pacific Northwest Laboratory is developing the IBS which will be released on July 1, 1992.

The CSEPP Emergency Management Information Functional Specification will provide the basis for the final CSEPP application on FEMIS.

\section{Extension to Other Emergencies}

The IBS provides the first baseline system for responding to CSEPP emergencies. It will be utilized in several locations and evaluated in terms of how well the system works and whether changes are required in any of the functional specifications. This feedback will be used to update the requirements for FEMIS/CSEPP.

When the requirements have been finalized, the design for FEMIS/CSEPP will be completed and the system will be developed. This system will replace the IBS and will undergo an operational evaluation that will provide the information necessary to complete the development of the common portion of FEMIS.

In order to extend this capability to the full spectrum of hazards, it will be necessary to couple the common portion of FEMIS with hazard-specific application managers and functional modules. This will provide a family of emergency support systems, e.g., FEMIS/Earthquake, FEMIS/Hurricane, etc. All of these systems will have a common "look and feel" to minimize training requirements and allow reuse of common response options, e.g., evacuation plans.

\section{SUMMARY}

Today's environment requires emergency managers, who face a wide spectrum of potential hazards, to make the proper decisions and take appropriate action in a very compressed time frame. Relying on traditional, manual response methods often does not allow the manager to consider the full range of available options and slows the response to the emergency, thereby placing the affected population in additional jeopardy. This problem can be solved by making better use of automation to process data and provide displays for decision makers that allow quick assimilation of the information..

FEMA is addressing this problem by developing an overall framework for integrating existing analytical tools and providing output in a form that is most appropriate, with special emphasis on the use of geographical displays. This framework will provide all of the human-computer interface and the functions that are common across the emergency spectrum and will integrate those applications and functions that are specific to a particular hazard. This approach will greatly reduce the time and effort required to learn the system, develop the necessary plans, and implement them in an emergency. It also allows current tools to be efficiently utilized in a wide spectrum of emergencies with little or no change, even if they were originally developed for use in one specific situation. 